\title{
ERGODICITY AND STABILITY OF NONSTATIONARY QUEUEING SYSTEMS
}

UDC 519.21

\author{
D. B. ANDREEV, M. A. ELESIN, E. A. KRYLOV, A. V. KUZNETSOV, AND A. I. ZEIFMAN
}

\begin{abstract}
We study stability and ergodicity of a special class of nonhomogeneous birth-death processes and consider applications of estimates for queue-length process for $M_{t} / M_{t} / S$ and $M_{t} / M_{t} / S / S$ queues.
\end{abstract}

\section{INTRODUCTION}

Nonstationary (nonhomogeneous in time) Markov chains have been studied over the last 20 years; see, for example, 11 14, 17, 3]. Nonstationary Markov chains appear, in particular, when describing queueing processes. Most of the research on those chains is devoted to various problems of their approximations (see [4, 19, 20] and references therein).

Important are the problems on the rate of convergence to a limit regime and on stability of nonstationary queueing systems. The first study on these problems was initiated by B. V. Gnedenko; see 8, 9, 10] and 15, 16.

The main tool of the present paper is the method used for the first time in [8] and developed in 25, 26, 27, 28, 29, 1, 12. The method is based on estimates using the log-norm of a linear operator function and on special transforms of the reduced operator function.

We apply this method to a class of Markov queueing systems with a special form of nonstationarity, which, however, is quite general for many applications.

\section{MAin Notation}

In what follows we consider a nonhomogeneous Markov process $X(t), t \geq 0$ (a queuelength process or a total number of customers arrived to a system), that is, a birth-death process with the phase space $E=\{0,1, \ldots, N\}, N \leq \infty$. It is assumed that the birth intensities $\lambda_{n}(t), t \geq 0$, and death intensities $\mu_{n}(t), t \geq 0, n \in E$, depend on time. Namely

$$
\mathrm{P}(X(t+h)=j / X(t)=i)= \begin{cases}\lambda_{i}(t) \cdot h+o(h) & \text { if } j=i+1, \\ \mu_{i}(t) \cdot h+o(h) & \text { if } j=i-1, \\ 1-\left(\lambda_{i}(t)+\mu_{i}(t)\right) \cdot h+o(h) & \text { if } j=i, \\ o(h) & \text { if }|i-j|>1 .\end{cases}
$$

Here $h>0$ and all symbols $o(h)$ are such that $o(h)=o(t, h) \rightarrow 0$ as $h \rightarrow 0, t \geq 0$, uniformly with respect to $i \in E$. Let

$$
p_{i j}(s, t)=\mathrm{P}(X(t)=j \mid X(s)=i), \quad i, j \in E, 0 \leq s \leq t,
$$

2000 Mathematics Subject Classification. Primary 60J27, 60J80. 
and $p_{i}(t)=\mathrm{P}(X(t)=i), i \in E, t \geq 0$, be transition probabilities and probabilities of states, respectively. Denote by $\mathbf{p}(t)=\left(p_{0}(t), \ldots, p_{N}(t)\right)^{T}, t \geq 0$, and by $A(t)=\left(a_{i j}(t)\right)$, $t \geq 0$, the vector column of probabilities of states, and the matrix of intensities related to (1). Note that

$$
a_{i j}(t)= \begin{cases}\lambda_{i-1}(t) & \text { if } j=i-1, \\ \mu_{i+1}(t) & \text { if } j=i+1, \\ -\left(\lambda_{i}(t)+\mu_{i}(t)\right) & \text { if } j=i, \\ 0 & \text { if }|i-j|>1 .\end{cases}
$$

Then the behavior of the process is described by the forward Kolmogorov system

$$
\frac{d \mathbf{p}}{d t}=A(t) \mathbf{p}, \quad \mathbf{p}=\mathbf{p}(t), \quad t \geq 0 .
$$

The Cauchy operator $U(t, s), 0 \leq s \leq t$, of equation (4) is determined by the matrix

$$
U^{T}(t, s)=P(s, t):=\left(p_{i j}(s, t)\right)_{i, j=0}^{N} .
$$

The norms of vectors and matrices used below are, as a rule, the $\ell_{1}$-norms denoted by $\|\cdot\|$, that is, $\|\mathbf{x}\|=\sum_{i \in E}\left|x_{i}\right|$ and

$$
\|B\|=\sup _{j \in E} \sum_{i \in E}\left|b_{i j}\right|
$$

for $\mathbf{x}=\left(x_{0}, \ldots, x_{N}\right)^{T}$ and $B=\left(b_{i j}\right)_{i, j=0}^{N}$, respectively.

The set of all stochastic vectors is denoted by $\Omega$ :

$$
\Omega=\left\{\mathbf{x}=\left(x_{0}, \ldots, x_{N}\right)^{T}: \mathbf{x} \geq 0,\|\mathbf{x}\|=1\right\} .
$$

\section{Stability}

Since

$$
p_{0}(t)=1-\sum_{i \geq 1} p_{i}(t), \quad t \geq 0,
$$

we obtain from (4) the system of differential equations:

$$
\frac{d \mathbf{z}}{d t}=B(t) \mathbf{z}(t)+\mathbf{f}(t), \quad t \geq 0,
$$

where

$$
\begin{aligned}
B(t) & =\left\{a_{i j}(t)-a_{i 0}(t), i, j=1, \ldots, N\right\}, \\
\mathbf{z}(t) & =\left(p_{1}(t), \ldots, p_{N}(t)\right)^{T}, \\
\mathbf{f}(t) & =\left(a_{10}(t), \ldots, a_{N 0}(t)\right)^{T}, \quad t \geq 0 .
\end{aligned}
$$

The solution of equation (6) is given by

$$
\mathbf{z}(t)=V(t, s) \cdot \mathbf{z}(s)+\int_{s}^{t} V(t, \tau) \cdot \mathbf{f}(\tau) d \tau, \quad 0 \leq s \leq t,
$$

where $V(t, s)$ is the Cauchy operator of system (6).

When study the stability and ergodicity, we often apply the inequality

$$
\left\|\mathbf{z}^{(1)}-\mathbf{z}^{(2)}\right\| \leq\left\|\mathbf{p}^{(1)}-\mathbf{p}^{(2)}\right\| \leq 2\left\|\mathbf{z}^{(1)}-\mathbf{z}^{(2)}\right\|, \quad t \geq 0,
$$

where

$$
\mathbf{z}^{(i)}=\mathbf{z}^{(i)}(t) \quad \text { and } \quad \mathbf{p}^{(i)}=\mathbf{p}^{(i)}(t), \quad t \geq 0, i=1,2,
$$

is an arbitrary pair of solutions of systems (6) and (4), respectively. 
The following transform:

$$
D=\left(\begin{array}{cccc}
d_{0} & d_{0} & d_{0} & \ldots \\
0 & d_{1} & d_{1} & \ldots \\
0 & 0 & d_{2} & \ldots \\
\ldots & \ldots & \ldots & \ldots
\end{array}\right),
$$

where $d_{i}>0, i=0,1, \ldots, N-1$, is introduced in 27, 28 in order to reduce the matrix $B(t)$ of system (6) to a more convenient form. Note that the transform is determined by an $N \times N$ upper triangle matrix. We have

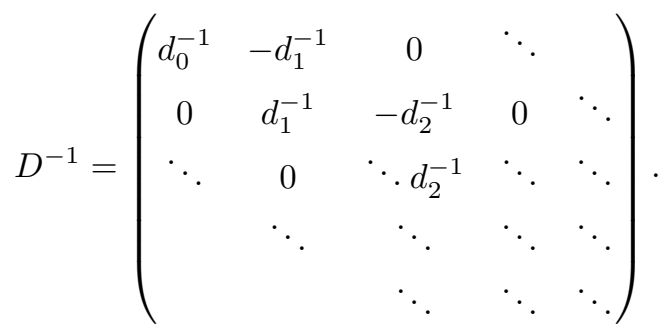

Applying this transform to the matrix $B(t)$ in (7) that corresponds to a nonhomogeneous birth-death process we get the matrix $D B(t) D^{-1}$ :

$$
D B(t) D^{-1}=\left(\begin{array}{ccccc}
-\left(\lambda_{0}(t)+\mu_{1}(t)\right) & d_{0} \cdot d_{1}^{-1} \cdot \mu_{1} & 0 & & \ldots \\
d_{1} \cdot d_{0}^{-1} \cdot \lambda_{1} & -\left(\lambda_{1}(t)+\mu_{2}(t)\right) & d_{1} \cdot d_{2}^{-1} \cdot \mu_{2} & 0 & \\
0 & d_{2} \cdot d_{1}^{-1} \cdot \lambda_{2}(t) & \ddots & \ddots & \ddots \\
\vdots & 0 & \ddots & \ddots & \ddots \\
& \ldots & \ddots & \ddots & \ddots
\end{array}\right) .
$$

Put $d_{0}=1$. For the case of a finite phase space we also put $d_{N}=0$. Let $\delta_{i+1}=d_{i+1} / d_{i}$, $i=0, \ldots, N-1$. Then the log-norm is given by

$$
\gamma(B(t))_{1 D}=\gamma\left(D B(t) D^{-1}\right)_{1}=-\inf _{k} \alpha_{k}(t)=-\underline{\alpha}(t), \quad t \geq 0
$$

(see, for example, [5, 29]), where

(13) $\alpha_{k}(t)=\lambda_{k}(t)+\mu_{k+1}(t)-\delta_{k+1} \cdot \lambda_{k+1}(t)-\delta_{k}^{-1} \cdot \mu_{k}(t), \quad t \geq 0, k=0, \ldots, N-1$.

Now we use the change $\mathbf{z}(t)=D^{-1} \mathbf{y}(t), t \geq 0$, and transform system (6) to a system with the matrix $D B(t) D^{-1}, t \geq 0$. As in [26, 27] we put

$$
\|\mathbf{z}\|_{1 D}=\|D \mathbf{z}\| \text {. }
$$

Then we get

$$
\|\mathbf{z}\|_{1 D} \geq \frac{g}{2}\|\mathbf{z}\|
$$

by (10), where $g=\inf _{k} d_{k}$. If the weights $\delta_{k}, k=0, \ldots, N-1$, in (13) are such that

$$
\int_{0}^{+\infty} \underline{\alpha}(t) d t=+\infty,
$$

then the birth-death process is weakly ergodic and

$$
\begin{gathered}
\left\|\mathbf{p}^{(1)}(t)-\mathbf{p}^{(2)}(t)\right\| \leq \frac{4}{g} \exp \left\{-\int_{s}^{t} \underline{\alpha}(u) d u\right\}\left\|\mathbf{z}^{(1)}(s)-\mathbf{z}^{(2)}(s)\right\|_{1 D}, \\
0 \leq s \leq t .
\end{gathered}
$$


Here

$$
\left\|\mathbf{z}^{(1)}(s)-\mathbf{z}^{(2)}(s)\right\|_{1 D} \leq \sum_{i \geq 1} q_{i}\left|p_{i}^{(1)}(s)-p_{i}^{(2)}(s)\right|, \quad t \geq 0
$$

according to notation (14), where $q_{i}=\sum_{m=0}^{i-1} d_{m}, i=1, \ldots, N$.

Estimate (16) holds for ergodic birth-death processes. This is seen by putting

$$
\mathbf{p}^{(2)}(t)=\boldsymbol{\pi}, \quad t \geq 0 .
$$

We consider a class of nonhomogeneous birth-death processes satisfying the condition

$$
\lambda_{n}(t)=\lambda_{n} \cdot a(t), \quad \mu_{n}(t)=\mu_{n} \cdot b(t), \quad t \geq 0, n \in E,
$$

where

$$
\mu_{0}=0, \quad \lambda_{n}>0, \quad n=0, \ldots, N-1, \quad \mu_{n}>0, \quad n=1, \ldots, N-1 .
$$

We assume that the following limits exist:

$$
\lim _{n \rightarrow \infty} \lambda_{n}=\lambda>0, \quad \lim _{n \rightarrow \infty} \mu_{n}=\mu>0
$$

if $N=\infty$. For the case of $N<\infty$ we put

$$
\lambda=\lambda_{N-1}, \quad \mu=\mu_{N} .
$$

We also assume that the functions $a(t) \geq 0, t \geq 0$, and $b(t) \geq 0, t \geq 0$, are locally integrable on $[0, \infty)$ and possess the "uniform" mean values

$$
\widetilde{a}=\lim _{t-s \rightarrow \infty} \frac{1}{t-s} \int_{s}^{t} a(\tau) d \tau, \quad \widetilde{b}=\lim _{t-s \rightarrow \infty} \frac{1}{t-s} \int_{s}^{t} b(\tau) d \tau .
$$

Condition (22) holds, in particular, if the functions $a(t)$ and $b(t)$ are periodic (in general, with different periods) or asymptotically periodic.

It is easy to show that condition (22) implies that the functions $a(t)$ and $b(t)$ are integral bounded on $[0, \infty)$, that is,

$$
\sup _{t \geq 0} \int_{t}^{t+1} a(\tau) d \tau=\mathrm{a}<\infty, \quad \sup _{t \geq 0} \int_{t}^{t+1} b(\tau) d \tau=\mathrm{b}<\infty .
$$

Note that the existence of ordinary means

$$
\lim _{t \rightarrow \infty} \frac{1}{t} \int_{0}^{t} a(\tau) d \tau, \quad \lim _{t \rightarrow \infty} \frac{1}{t} \int_{0}^{t} b(\tau) d \tau
$$

does not imply the integral boundedness of $a(t)$ and $b(t)$.

In the current paper we study the stability of birth-death processes with respect to the perturbations of a more general form as compared to those in 25, 26, 27, 30, 12,

We assume that the perturbed Markov chain $\bar{X}(t), t \geq 0$, is a process with bounded jumps, that is, its matrix of intensities

$$
\bar{A}(t)=\left(\bar{a}_{i j}(t)\right)_{i, j=0}^{N}
$$

is such that $\bar{a}_{i j}(t)=0$ for all $t \geq 0,|i-j|>R$, and some positive integer $R$. Moreover we assume that

$$
\bar{a}_{i j}(t)=\bar{a}_{i j} \bar{a}(t)
$$

for $i>j$, while

$$
\bar{a}_{i j}(t)=\bar{a}_{i j} \bar{b}(t)
$$


for $i<j$, and

$$
\begin{gathered}
L=\sup _{i, j, i \neq j}\left(a_{i j}, \bar{a}_{i j}\right)<\infty, \\
\sup _{t \geq 0} \int_{t}^{t+1}(|\hat{a}(\tau)|+|\hat{b}(\tau)|) d \tau \leq \varepsilon,
\end{gathered}
$$

where $\hat{a}(t)=a(t)-\bar{a}(t)$ and $\hat{b}(t)=b(t)-\bar{b}(t)$.

Put

$$
\begin{gathered}
f_{k}=\frac{\Delta \lambda \mu_{k+1}-\lambda_{k-1} \mu+\sqrt{\left(\Delta \lambda \mu_{k+1}-\lambda_{k-1} \mu\right)^{2}+4 \Delta \lambda \mu \cdot\left(\lambda_{k-1} \mu_{k+1}-\lambda_{k} \mu_{k}\right)}}{2 \Delta \lambda \mu}, \\
k=0, \ldots, N-1,
\end{gathered}
$$

where $\Delta>0$ and $\lambda$ and $\mu$ are defined by (20) and (21), respectively.

In what follows we use the notation

$$
\tilde{E}= \begin{cases}\{0,1, \ldots, N-1\} & \text { if } N<\infty \\ E & \text { if } N=\infty\end{cases}
$$

Put

$$
c_{0}=\min \left(1, \inf _{k} f_{k}, \inf _{k} \frac{\mu_{k+1}}{\mu}\right) .
$$

It is proved in [12] that for every $0<c \leq c_{0}$ there exists a positive sequence $\left\{\delta_{k}\right\}$ such that

$$
\underline{\alpha}(t) \geq l(t)=c \cdot(\mu b(t)-\Delta \lambda a(t)), \quad t \geq 0,
$$

for some $\Delta>1$ if $\inf _{k} f_{k}>0$. Moreover $\liminf \delta_{k}>1$ for $N=\infty$ and

$$
0<\mathrm{m}_{1} \leq \frac{\mu_{k}}{\mu_{k+1}-c \mu} \leq \delta_{k} \leq \frac{\lambda_{k-1}+c \Delta \lambda}{\lambda_{k}} \leq \mathrm{m}_{2}<\infty
$$

under the latter condition.

Conditions (22) and (28) imply that there exists a constant $K$ such that

$$
\exp \left(-\int_{s}^{t} l(u) d u\right) \leq K \exp (-l(t-s))
$$

for all $0 \leq s \leq t$, where

$$
l=c(\mu \widetilde{b}-\Delta \lambda \widetilde{a})-\varepsilon
$$

Let

$$
\mu \widetilde{b}-\Delta \lambda \widetilde{a}>0, \quad \mathbf{p}(0)=\overline{\mathbf{p}}(0),
$$

and the "perturbed" Markov chain $\bar{X}(t), t \geq 0$, has bounded jumps and satisfies conditions (24) and (25).

We rewrite condition (6) as follows:

$$
\begin{aligned}
\frac{d \mathbf{z}}{d t} & =\bar{B}(t) \mathbf{z}+\overline{\mathbf{f}}(t)+(B(t)-\bar{B}(t)) \mathbf{z}+\mathbf{f}(t)-\overline{\mathbf{f}}(t) \\
& =\bar{B}(t) \mathbf{z}+\overline{\mathbf{f}}(t)+\hat{B}(t) \mathbf{z}+\hat{\mathbf{f}}(t),
\end{aligned}
$$

where $\hat{B}(t)=B(t)-\bar{B}(t)$ and $\hat{\mathbf{f}}(t)=\mathbf{f}(t)-\overline{\mathbf{f}}(t), t \geq 0$. Then

$$
\begin{aligned}
\mathbf{z}(t) & =\bar{V}(t, 0) \cdot \mathbf{z}(0)+\int_{0}^{t} \bar{V}(t, \tau) \cdot \overline{\mathbf{f}}(\tau) d \tau+\int_{0}^{t} \bar{V}(t, \tau) \cdot[\hat{B}(\tau) \mathbf{z}(\tau)+\hat{\mathbf{f}}(\tau)] d \tau \\
& =\overline{\mathbf{z}}(t)+\int_{0}^{t} \bar{V}(t, \tau) \cdot[\hat{B}(\tau) \mathbf{z}(\tau)+\hat{\mathbf{f}}(\tau)] d \tau, \quad t \geq 0,
\end{aligned}
$$


and we get

$$
\begin{aligned}
\|\mathbf{z}(t)-\overline{\mathbf{z}}(t)\|_{1 D} & \leq \int_{0}^{t}\|\bar{V}(t, \tau)\|_{1 D} \cdot\|\hat{B}(\tau) \mathbf{z}(\tau)+\hat{\mathbf{f}}(\tau)\|_{1 D} d \tau \\
& \leq \int_{0}^{t}\|\bar{V}(t, \tau)\|_{1 D} \cdot\left\{\|\hat{B}(\tau)\|_{1 D} \cdot\|\mathbf{z}(\tau)\|_{1 D}+\|\hat{\mathbf{f}}(\tau)\|_{1 D}\right\} d \tau
\end{aligned}
$$

According to (30) and (23) we have

$$
\begin{aligned}
\|\mathbf{z}(t)\|_{1 D} & \leq\|V(t, 0)\| \cdot\|\mathbf{z}(0)\|_{1 D}+\int_{0}^{t}\|V(t, \tau)\|_{1 D} \cdot\|\mathbf{f}(\tau)\|_{1 D} d \tau \\
& \leq K e^{-l t}\|\mathbf{z}(0)\|_{1 D}+\lambda_{0} e^{-l t} \int_{0}^{t} e^{l \tau} a(\tau) d \tau \\
& \leq K e^{-l t}\|\mathbf{z}(0)\|_{1 D}+\lambda_{0} e^{-l t}\left(\sum_{n=0}^{[t]-1} \int_{n}^{n+1} e^{l \tau} a(\tau) d \tau+\int_{[t]}^{t} e^{l \tau} a(\tau) d \tau\right) \\
& \leq K e^{-l t}\|\mathbf{z}(0)\|_{1 D}+\lambda_{0} \mathrm{a}\left(1+\frac{e^{l}}{e^{l}-1}\right) .
\end{aligned}
$$

Let $\hat{a}_{i j}(t)=a_{i j}(t)-\bar{a}_{i j}(t)$. Then

$$
D \hat{B} D^{-1}=\left(\begin{array}{ccc}
g_{11} & g_{12} & \ldots \\
g_{21} & g_{22} & \ldots \\
\ldots & \ldots & \ldots
\end{array}\right)
$$

where

$$
\begin{aligned}
& g_{11}=\hat{a}_{11}+\hat{a}_{21}+\cdots-\hat{a}_{10}-\hat{a}_{20}-\cdots, \\
& g_{12}=d_{0} d_{1}^{-1}\left(\hat{a}_{12}+\hat{a}_{22}+\cdots-\hat{a}_{11}-\hat{a}_{21}-\cdots\right), \\
& g_{21}=d_{1} d_{0}^{-1}\left(\hat{a}_{21}+\hat{a}_{31}+\cdots-\hat{a}_{20}-\hat{a}_{30}-\cdots\right), \\
& g_{22}=\left(\hat{a}_{22}+\hat{a}_{32}+\cdots-\hat{a}_{21}-\hat{a}_{31}-\cdots\right) .
\end{aligned}
$$

Since the jumps are bounded, the entries of the above matrix are zero if the absolute value of the difference of indices is greater than $R$. Putting

$$
\mathrm{m}_{3}=\left(\max \left(\mathrm{m}_{2}, \mathrm{~m}_{1}^{-1}\right)\right)^{R},
$$

we get

$$
\begin{gathered}
\|\hat{B}(t)\|_{1 D}=\left\|D \hat{B}(t) D^{-1}\right\|_{1} \leq 2 L R \mathrm{~m}_{3}(|\hat{a}(t)|+|\hat{b}(t)|), \\
\|\hat{\mathbf{f}}(t)\|_{1 D} \leq L R \mathrm{~m}_{3}|\hat{a}(t)|
\end{gathered}
$$

and

$$
\gamma(\bar{B}(t))_{1 D} \leq \gamma(B(t))_{1 D}+\|\hat{B}(t)\| \leq-l(t)+2 L R \mathrm{~m}_{3}(|\hat{a}(t)|+|\hat{b}(t)|) .
$$

The last inequality implies that

$$
\begin{aligned}
\|\bar{V}(t, \tau)\| & \leq K \exp \left(-l(t-\tau)+\int_{\tau}^{t} 2 L R \mathrm{~m}_{3}(|\hat{a}(\tau)|+|\hat{b}(\tau)|) d \tau\right) \\
& \leq 2 K \exp \left\{-\left(l-2 L R \mathrm{~m}_{3} \varepsilon\right)(t-\tau)\right\}
\end{aligned}
$$


by (24) and (25). Thus

$$
\begin{aligned}
\|\mathbf{p}(t)-\overline{\mathbf{p}}(t)\|_{1} \leq & \frac{4}{g}\|\mathbf{z}(t)-\overline{\mathbf{z}}(t)\|_{1 D} \\
\leq & \frac{8}{g} K L R \mathrm{~m}_{3} \int_{0}^{t} \exp \left\{-\left(l-2 L R \mathrm{~m}_{3} \varepsilon\right)(t-u)\right\}(|\hat{a}(u)|+|\hat{b}(u)|) \\
\quad & \quad \times\left(2 K\|\mathbf{z}(0)\|_{1 D}+2 \lambda_{0} \mathrm{a}\left(1+\frac{e^{l}}{e^{l}-1}\right)+1\right) d u \\
\leq & \frac{16}{g} \varepsilon K L R \mathrm{~m}_{3}\left(2 K\|\mathbf{z}(0)\|_{1 D}+2 \lambda_{0} \mathrm{a}\left(1+\frac{e^{l}}{e^{l}-1}\right)+1\right) \\
& \times\left(1+\frac{e^{\left(l-2 L R \mathrm{~m}_{3} \varepsilon\right)}}{e^{\left(l-2 L R \mathrm{~m}_{3} \varepsilon\right)}-1}\right) .
\end{aligned}
$$

Therefore the following result holds.

Theorem 1. Assume that condition (31) holds and the "perturbed" Markov chain $\bar{X}(t)$, $t \geq 0$, has bounded jumps and satisfies conditions (24) and (25). Then estimate (40) holds.

Now we consider the case of a finite phase space. The equation for the "perturbed" chain, similar to [6], can be written as follows:

$$
\frac{d \overline{\mathbf{z}}}{d t}=B(t) \overline{\mathbf{z}}+\mathbf{f}(t)+(\bar{B}(t)-B(t)) \overline{\mathbf{z}}+\overline{\mathbf{f}}(t)-\mathbf{f}(t)=B(t) \overline{\mathbf{z}}+\mathbf{f}(t)-\hat{B}(t) \overline{\mathbf{z}}-\hat{\mathbf{f}}(t) .
$$

Then

$$
\begin{gathered}
\|\overline{\mathbf{z}}(t)\|_{1} \leq\|\overline{\mathbf{p}}(t)\|_{1} \leq 1, \\
\|\hat{B}(t)\|_{1} \leq 2 L(|\hat{a}(t)|+|\hat{b}(t)|), \\
\|\hat{\mathbf{f}}(t)\|_{1} \leq L|\hat{a}(t)|, \\
\|V(t, \tau)\|_{1} \leq\|D\| \cdot\left\|D^{-1}\right\| \cdot \exp \left\{-\int_{s}^{t} l(u) d u\right\} \leq N G \cdot \frac{2}{g} \cdot K e^{-l(t-s)},
\end{gathered}
$$

where $G=\max d_{i}$.

Thus for the $\ell_{1}$-norm we get

$$
\begin{aligned}
\|\mathbf{z}(t)-\overline{\mathbf{z}}(t)\| & \leq \int_{0}^{t}\|V(t, \tau)\| \cdot\|\hat{B}(\tau) \overline{\mathbf{z}}(\tau)+\hat{\mathbf{f}}(\tau)\| d \tau \\
& \leq \int_{0}^{t}\|V(t, \tau)\| \cdot\{\|\hat{B}(\tau)\| \cdot\|\overline{\mathbf{z}}(\tau)\|+\|\hat{\mathbf{f}}(\tau)\|\} d \tau \\
& \leq \frac{6 G N K L}{g} \cdot \int_{0}^{t} e^{-l(t-\tau)}(|\hat{a}(\tau)|+|\hat{b}(\tau)|) d \tau \\
& \leq \frac{6 G N K L}{g} \cdot \varepsilon\left(1+\frac{e^{l}}{e^{l}-1}\right) .
\end{aligned}
$$

Therefore the following result holds.

Theorem 2. Let all the assumptions of Theorem 1 hold and $N<\infty$. If the intensities of the "perturbed" Markov chain $\bar{X}(t), t \geq 0$, satisfy conditions (24) and (25), then

$$
\|\mathbf{p}(t)-\overline{\mathbf{p}}(t)\|_{1} \leq \frac{12 G N K L \varepsilon}{g}\left(1+\frac{e^{l}}{e^{l}-1}\right) .
$$




\section{EXAMPLES}

First we consider a queueing system close to $M_{t} / M_{t} / S$. The corresponding references for the system $M_{t} / M_{t} / S$ can be found in [13, 21, 22, 2]. We assume that the number of customers in the queue (the queue length) is a birth-death process $X(t)$ whose intensities are locally integrable on $[0, \infty)$ :

$$
\lambda_{n}(t)=a(t), \quad \mu_{n}(t)=b(t) \cdot \min (n, S) .
$$

The stability of these processes is studied in [29, 12]. Assume that the uniform means $\widetilde{a}$ and $\widetilde{b}$ exist. We also assume that the traffic intensity

$$
\rho=\frac{\widetilde{a}}{S \widetilde{b}}
$$

is such that $(1-1 / S)^{2} \leq \rho<1$. Then

$$
c_{0}=\min \left(\frac{1}{S}, \frac{\Delta-1}{\Delta}\right)=\frac{\Delta-1}{\Delta}=1-\rho^{1 / 2},
$$

$\delta_{k}=\rho^{-1 / 2}>1$, and

$$
l(t)=\left(1-\rho^{1 / 2}\right)\left(S b(t)-\rho^{-1} a(t)\right) .
$$

Note that estimate (49) is quite precise, since the true value of the convergence parameter for a stationary queueing system $M / M / S$ is equal to

$$
\dot{\alpha}=(\sqrt{a}-\sqrt{S b})^{2}
$$

(see [6]).

Now we assume that a queueing system is close to $M_{t} / M_{t} / S$ in the sense that customers may arrive to the system and be served in groups. The number of customers in every group does not exceed $R$ and the perturbations of arrival and service intensities of customers satisfy conditions (24) and (25).

Then the following estimate of the "stability" holds:

$$
\begin{aligned}
\|\mathbf{p}(t)-\overline{\mathbf{p}}(t)\|_{1} \leq & 16 \varepsilon K L R \mathrm{~m}_{3}\left(2 K\|\mathbf{z}(0)\|_{1 D}+2 \mathrm{a}\left(1+\frac{e^{l}}{e^{l}-1}\right)+1\right) \\
& \times\left(1+\frac{e^{\left(l-2 L R \mathrm{~m}_{3} \varepsilon\right)}}{e^{\left(l-2 L R \mathrm{~m}_{3} \varepsilon\right)}-1}\right)
\end{aligned}
$$

where $\mathrm{m}_{3}=\rho^{-R / 2}$. If there are $H$ customers in the system at the moment $t=0$, then

$$
\|\mathbf{z}(0)\|_{1 D} \leq \sum_{k=0}^{H} \rho^{-k / 2} .
$$

It is worthwhile to mention that if the arrival and service intensities of customers are asymptotically periodic, then the corresponding process is "almost ergodic" in the sense that the limit

$$
\tilde{p}_{\infty}=\lim _{t \rightarrow \infty} \frac{1}{t} \int_{0}^{t} \mathbf{p}(\tau) d \tau
$$

exists and is independent of the initial probability distribution of states.

Now we consider queueing systems close to $M_{t} / M_{t} / S / 0$.

There is a number of papers devoted to the queueing system $M_{t} / M_{t} / S / 0$ (the system with losses). Usually it is assumed that the intensities are constant (see references in [18] 
and follow up papers [7, 24, 23]). The number of customers $X(t)$ for this queue is a birth-death process with a finite phase space $E=\{0,1, \ldots, S\}$ and with intensities

$$
\lambda_{n}(t)=a(t), \quad \mu_{n}(t)=n b(t) .
$$

The first results and estimates for the general case are obtained in [27]. (also see [29] 12]). Some of the results of 27] are obtained in a follow-up paper 20], where the nonMarkovian case is also studied. We assume again that the uniform means $\widetilde{a}$ and $\widetilde{b}$ exist and moreover $\widetilde{b}>0$. Putting $\delta_{k}=1$ for all $k$ we prove that $\alpha_{k}(t) \geq b(t)$ for all $k$ and

$$
l(t)=b(t) .
$$

Let a queueing system be close to $M_{t} / M_{t} / S / 0$ in the sense that the total number of customers in the system does not exceed $S$ and the perturbations of the arrival and service intensities satisfy conditions (24) and (25)).

Then Theorem 1 implies the following estimate of the "stability":

$$
\begin{aligned}
\| \mathbf{p}(t) & -\overline{\mathbf{p}}(t) \|_{1} \\
& \leq 16 \varepsilon K L S\left(2 K S+2 \mathrm{a}\left(1+\frac{e^{l}}{e^{l}-1}\right)+1\right)\left(1+\frac{e^{\left(l-2 L R \mathrm{~m}_{3} \varepsilon\right)}}{e^{\left(l-2 L R \mathrm{~m}_{3} \varepsilon\right)}-1}\right) .
\end{aligned}
$$

Theorem 2 implies a sharper estimate, which is, naturally, of the same order:

$$
\|\mathbf{p}(t)-\overline{\mathbf{p}}(t)\|_{1} \leq 12 \varepsilon K L S\left(1+\frac{e^{l}}{e^{l}-1}\right) .
$$

In the case of asymptotically periodic arrival and service intensities, the corresponding process is "almost ergodic" in the sense that the limit

$$
\tilde{p}_{\infty}=\lim _{t \rightarrow \infty} \frac{1}{t} \int_{0}^{t} \mathbf{p}(\tau) d \tau
$$

exists and is independent of the initial probability distribution of customers. Note that the limit above can be used as the "pseudostationary distribution".

\section{BIBLIOGRAPHY}

1. D. B. Andreev, M. A. Elesin, E. A. Krylov, A. V. Kuznetsov, and A. I. Zeifman, On ergodicity and stability estimates for some nonhomogeneous Markov chains, J. Math. Sci. 112 (2002), 4111-4118. MR 2003m:60204

2. J. R. Artalejo and M. J. Lopez-Herrero, Analysis of the busy period for the $M / M / c$ queue: an algorithmic approach, J. Appl. Prob. 38 (2001), 209-222. MR 2001k:60130

3. J.-D. Deuschel and C. Mazza, $L^{2}$ convergence of time nonhomogeneous Markov processes: I. Spectral estimates, Ann. Appl. Prob. 4 (1994), no. 4, 1012-1056. MR 96b:60188

4. A. Di Crescenzo and A. G. Nobile, Diffusion approximation to a queueing system with time dependent arrival and service rates, QUESTA 19 (1995), 41-62. MR 96b:60238

5. Yu. L. Daletskiǔ and M. G. Krein, Stability of Solutions of Differential Equations in Banach Spaces, "Nauka", Moscow, 1970; English transl., Amer. Math. Soc., Providence, RI, 1974. MR 50:5125; MR 50:5126

6. van E. Doorn, Conditions for exponential ergodicity and bounds for the decay parameter of a birth-death process, Adv. Appl. Prob. 17 (1985), 504-530. MR 86m:60183

7. C. Fricker, P. Robert, and D. Tibi, On the rate of convergence of Erlang's model, J. Appl. Prob. 36 (1999), 1167-1184. MR 2000k:60186

8. B. V. Gnedenko and I. P. Makarov, Properties of solutions of a problem with losses in the case of periodic intensities, Differentsial'nye Uravneniya 7 (1971), 1696-1698. (Russian) MR 45:2254

9. B. V. Gnedenko and A. D. Solov'ev, On conditions of the existence of final probabilities for a Markov process, Math. Operationsforsch. Statist. 4 (1973), 379-390. (Russian) MR 52:15680

10. B. V. Gnedenko, On a generalization of Erlang's formulae, Zastos. Mat. 12 (1971), 239-242. (Russian) MR 47:4351 
11. V. Giorno and A. Nobile, On some time-nonhomogeneous diffusion approximations to queueing systems, Adv. Appl. Prob. 19 (1987), 974-994. MR 89a:60218

12. B. L. Granovsky and A. I. Zeifman, Nonstationary Markovian queues, J. Math. Sci. 99 (2000), no. 4, 1415-1438. MR 2001g:60206

13. L. Green, P. Kolesar, and A. Svornos, Some effects of nonstationarity on multiserver Markovian queueing systems, Oper. Res. 39 (1991), 502-511.

14. D. P. Heyman and W. Whitt, The asymptotic behaviour of queues with time-varying arrival rates, J. Appl. Prob. 21 (1984), 143-156. MR 85c:60158

15. V. V. Kalashnikov, Qualitative analysis of complex systems behavior by the test functions method, "Nauka", Moscow, 1978. (Russian) MR 82b:90041

16. N. V. Kartashov, Strong stable Markov chains, Problems of the Stability for Stochastic Models, VNIISI, Moscow, 1981, pp. 54-59; English transl., J. Soviet Math. 34 (1986), 1493-1498. MR 84b:60089

17. J. B. Keller, Time-dependent queues, SIAM Rev. 24 (1982), 401-412. MR 85c:60160

18. M. Kijima, On the largest negative eigenvalue of the infinitesimal generator associated with $M / M / n / n$ queues, Oper. Res. Let. 9 (1990), 59-64. MR 91f:60171

19. A. Mandelbaum and W. Massey, Strong approximations for time-dependent queues, Math. Oper. Res. 20 (1995), 33-64. MR 96b:60240

20. W. A. Massey and W. Whitt, On analysis of the modified offered-load approximation for the nonstationary Erlang loss model, Ann. Appl. Prob. 4 (1994), 1145-1160. MR 95m:60147

21. M. H. Rothkopf and S. S. Oren, A closure approximation for the nonstationary $M / M / s$ queue, Management Sci. 25 (1979), 522-534. MR 81c:60101

22. W. Stadie and P. R. Parthasarathy, On the convergence to stationarity of the many-server Poisson queue, J. Appl. Prob. 36 (1999), 546-557. MR 2000i:60109

23. W. Stadie and P. R. Parthasarathy, Generating function analysis of some joint distributions for Poisson loss systems, QUESTA 34 (2000), 183-197. MR 2001g:60238

24. M. Voit, A note of the rate of convergence to equilibrium for Erlang's model in the subcritical case, J. Appl. Prob. 37 (2000), 918-923. MR 2001e:60183

25. A. I. Zeifman, Stability for continuous-time nonhomogeneous Markov chains, Lect. Notes Math. 1155 (1985), 401-414. MR 87h:60136

26. A. I. Zeifman, Qualitative properties of nonhomogeneous birth-death processes, Problems of the Stability for Stochastic Models, VNIISI, Moscow, 1988, pp. 32-40; English transl., J. Soviet Math. 57 (1991), 3217-3224. MR 92b:60084

27. A. I. Zeifman, Properties of a loss system in the case of variable rate, Avtomat. i Telemekh. 1 (1989), 107-113; English transl., Automat. Remote Control 50 (1989), 82-87. MR 90g:60088

28. A. I. Zeifman, Some estimates of the rate of convergence for birth and death processes, J. Appl. Prob. 28 (1991), 268-277. MR 92f:60147

29. A. I. Zeifman, Upper and lower bounds on the rate of convergence for nonhomogeneous birth and death processes, Stoch. Proc. Appl. 59 (1995), 157-173. MR 96g:60109

30. A. I. Zeifman and D. Isaacson, On strong ergodicity for nonhomogeneous continuous-time Markov chains, Stoch. Proc. Appl. 50 (1994), 263-273. MR 95c:60068

Vologda State Pedagogical University, Vologda, Russia

Vologda State Pedagogical University, Vologda, Russia

Vologda State Pedagogical University, Vologda, Russia

Vologda State Pedagogical University, Vologda, Russia

Vologda State Pedagogical University, Vologda, Russia

Current address: Vologda Scientific Coordinate Centre of Central Economics and Mathematics Institute, Russian Academy of Sciences, Vologda, Russia

E-mail address: zai@uni-vologda.ac.ru

Received 4/APR/2002

Translated by THE AUTHORS 